\title{
Propofol Restores the Function of "Hyperekplexic" Mutant Glycine Receptors in Xenopus Oocytes and Mice
}

\author{
Sean Michael 0'Shea, ${ }^{1}$ Lore Becker, ${ }^{2}$ Hans Weiher, ${ }^{2}$ Heinrich Betz, ${ }^{1}$ and Bodo Laube ${ }^{1}$ \\ ${ }^{1}$ Abteilung Neurochemie, Max-Planck-Institut für Hirnforschung, 60528 Frankfurt am Main, Germany, and Institute for Diabetes Research, \\ 80804 Munich, Germany
}

\begin{abstract}
Human hereditary hyperekplexia ("startle disease") is a neurological disorder characterized by exaggerated, convulsive movements in response to unexpected stimuli. Molecular genetic studies have shown that this disease is often caused by amino acid substitutions at arginine 271 to glutamine or leucine of the $\alpha_{1}$ subunit of the inhibitory glycine receptor (GlyR). When exogenously expressed in Xenopus oocytes, agonist responses of mutant $\alpha_{1}(\mathrm{R} 271 \mathrm{Q})$ and $\alpha_{1}(\mathrm{R} 271 \mathrm{~L})$ GlyRs show higher $\mathrm{EC}_{50}$ values and lower maximal inducible responses (relative efficacies) compared with oocytes expressing wild-type $\alpha_{1}$ GlyR subunits. Here, we report that the maximal glycine-induced currents $\left(I_{\max }\right)$ of mutant $\alpha_{1}(\mathrm{R} 271 \mathrm{Q})$ and $\alpha_{1}(\mathrm{R} 271 \mathrm{~L})$ GlyRs were dramatically potentiated in the presence of the anesthetic propofol (PR0), whereas the $I_{\max }$ of wild-type $\alpha_{1}$ receptors was not affected. Quantitative analysis of the agonist responses of the isofunctionally substituted $\alpha_{1}(\mathrm{R} 271 \mathrm{~K})$ mutant GlyR revealed that saturating concentrations of PRO decreased the $\mathrm{EC}_{50}$ values of both glycine and the partial agonist $\beta$-alanine by $>10$-fold, with relative efficacies increasing by 4 - and 16-fold, respectively. Transgenic (tg) mice carrying the $\alpha_{1}(\mathrm{R} 271 \mathrm{Q})$ mutation $(\operatorname{tg} 271 \mathrm{Q}-300)$ have both spontaneous and induced tremor episodes that closely resemble the movements of startled hyperekplexic patients. After treatment with subanesthetic doses of PRO, the tg271Q-300 mutant mice showed temporary reflexive and locomotor improvements that made them indistinguishable from wild-type mice. Together, these results demonstrate that the functional and behavioral effects of hyperekplexia mutations can be effectively reversed by drugs that potentiate GlyR responses.
\end{abstract}

Key words: anesthesia; glycine; inhibition; IPSP (inhibitory potential); receptor; startle

\section{Introduction}

Patients affected by hyperekplexia, a neurological disorder commonly known as "startle disease," reflexively jump, yell, or extend their arms in response to an unexpected touch or sound (Andermann et al., 1980). In severe cases, a mild stimulus produces stereotypic, convulsive jerks that resemble the effects of strychnine poisoning (Floeter and Hallet, 1993). In infants, the condition is often termed "stiff baby syndrome," because a light touch to the tip of the nose can cause a muscle rigidity that temporarily halts breathing and may even lead to death caused by apnea (Stewart et al., 2002). Hyperekplexia is hereditary, and genetic linkage studies of affected patients have traced the disease to at least seven naturally occurring point mutations in glycine receptor (GlyR) $\alpha_{1}$ subunits (Schofield, 2002).

The most commonly observed hyperekplexia mutations are R271L and R271Q, found in the extracellular M2-M3 linker region of the $\alpha_{1}$ GlyR subunit (Shiang et al., 1993). When heterologously expressed, homo-oligomeric GlyRs containing $\alpha_{1}(\mathrm{R} 271)$

\footnotetext{
Received 0ct. 16, 2003; revised Jan. 13, 2004; accepted Jan. 14, 2004.

This work was supported by a fellowship from the Max-Planck-Gesellschaft (S.M.O.), Bundesministerium für Bildung und Forschung (H.W., H.B.), Deutsche Forschungsgemeinschaft Grant LA 1086/2-2 (B.L.), Fritz Thyssen Stiftung (H.W.), and Fonds der Chemischen Industrie (H.B.)

Correspondence should be addressed to Dr. Bodo Laube, Max-Planck-Institut für Hirnforschung, Abteilung Neurochemie, Deutschordentrasse 46, 60528 Frankfurt am Main, Germany. E-mail: laube@mpih-frankfurt.mpg.de.

S. M. O'Shea's present address: Auerbach Laboratory, Department of Physiology and Biophysics, 309 Cary Hall, State University of New York at Buffalo, Buffalo, NY 14214.

DOI:10.1523/JNEUROSCI.4675-03.2004

Copyright $\odot 2004$ Society for Neuroscience $\quad$ 0270-6474/04/242322-06\$15.00/0
}

substitutions show enormous reductions in agonist sensitivity and maximal inducible currents (Langosch et al., 1994). The isofunctional substitution $\mathrm{R} 271 \mathrm{~K}$ also increases the glycine $\mathrm{EC}_{50}$ value, but to a lesser extent than the natural occurring mutants (Langosch et al., 1994). Similarly, most $\alpha_{1}$ (R271) substitutions increase the $\mathrm{EC}_{50}$ values and reduce the maximal responses of the partial agonists $\beta$-alanine and taurine (Laube et al., 1995).

Based on these findings, we recently developed an animal model for hyperekplexia by expressing human $\alpha_{1}(\mathrm{R} 271 \mathrm{Q}) \mathrm{GlyR}$ subunits in a transgenic (tg) mouse line (Becker et al., 2002). In these tg271Q-300 mice, mutant $\alpha_{1}$ subunits co-assemble with wild-type (wt) $\alpha_{1}$ subunits to reduce GlyR agonist sensitivity without decreasing GlyR surface expression (Becker et al., 2002). Behaviorally, tg271Q-300 mice have uncoordinated, spastic movements that lead to significantly slower righting times $(>10$ $\mathrm{sec})$ than seen with wt mice $(<1 \mathrm{sec})$. In response to a sudden noise or touch, mutant animals show uncontrolled jumps and jerks that closely resemble the startle reflexes of hyperekplexia patients. On more vigorous handling, the mice display tremor episodes that are especially pronounced in the limbs. Because the amplitudes of glycinergic IPSCs recorded from spinal cords of tg271Q-300 mice are threefold smaller than the IPSCs of wt mice, there is now direct evidence that hereditary hyperekplexia is caused by a reduction in GlyR function.

Here, we report that the intravenous anesthetic propofol (PRO) can compensate functionally for reduced GlyR response amplitudes in two model systems for hyperekplexia. In oocytes, 

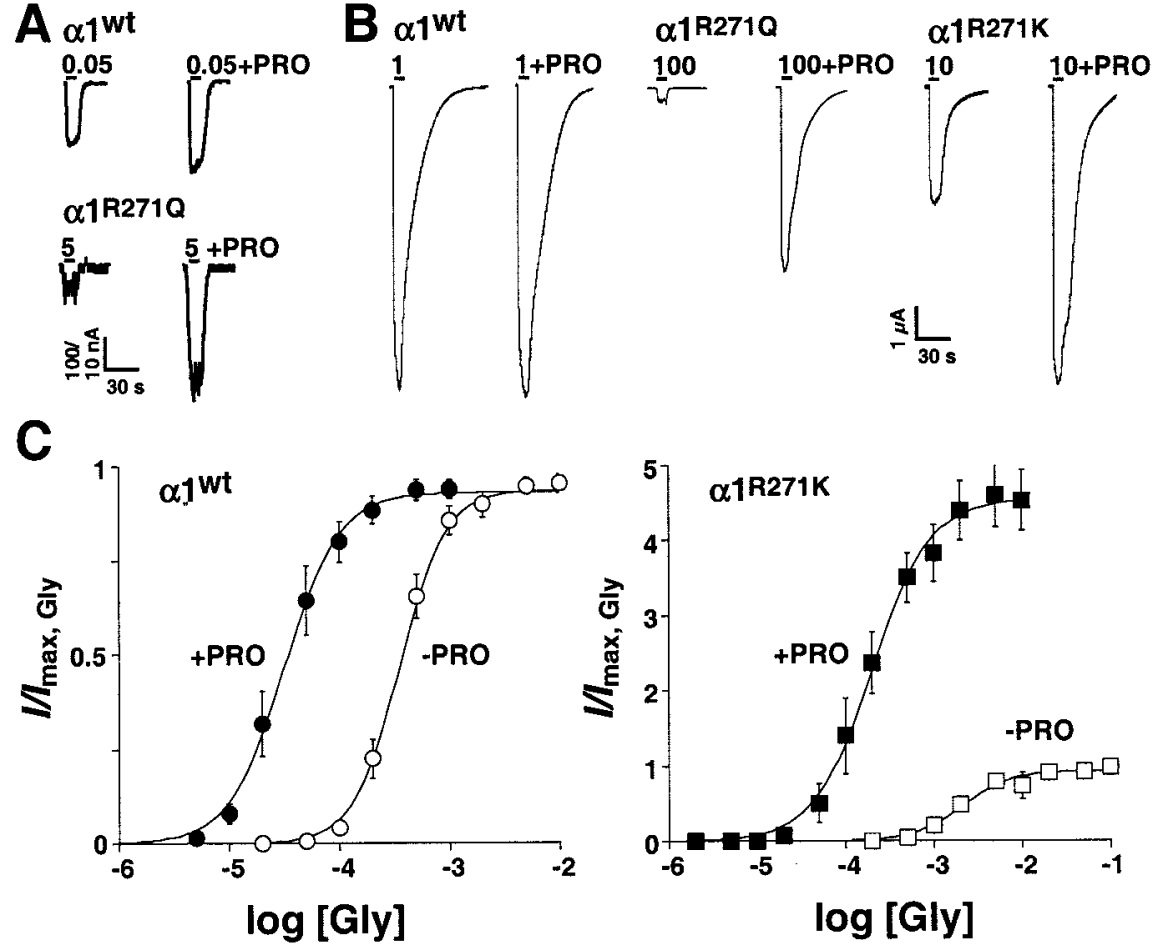

Figure 1. PRO enhances the maximal responses of hyperekplexic mutant GlyRs to glycine. A, Agonist-induced responses from oocytes expressing, wt $\alpha_{1}$ or $\alpha_{1}$ (R271Q) GlyRs in the absence (left traces) or presence (right traces) of bath-applied PRO (1 $\mu \mathrm{M}$ ) in the presence of submaximal glycine concentrations. Bars indicate the duration of glycine application; glycine concentrations are given in mm. B, Agonist-induced responses from oocytes expressing, from left, wt $\alpha_{1}, \alpha_{1}(\mathrm{R} 2710)$, or $\alpha_{1}(\mathrm{R} 271 \mathrm{~K})$ GlyRs in the absence (left traces) or presence (right traces) of bath-applied PRO $(0.5 \mathrm{~mm})$ at saturating glycine concentrations. Bars indicate the duration of glycine application; glycine concentrations are given in $\mathrm{mm}$. C, Dose-response curves for glycine in the absence (open circles) and presence (filled circles) of PRO (0.5 mM) for wt $\alpha_{1}$ and $\alpha_{1}$ (R271K) GlyRs. Data points represent means from six experiments, normalized to $I_{\max , \text { Gly }}$ obtained with either 1 or $100 \mathrm{~mm}$ glycine. Error bars (shown when larger than symbols) indicate SEM.

PRO increases the maximal responses to agonists in $\alpha_{1}(\mathrm{R} 271 \mathrm{Q})$, $\alpha_{1}(\mathrm{R} 271 \mathrm{~L})$, and $\alpha_{1}(\mathrm{R} 271 \mathrm{~K})$ GlyRs expressed in oocytes. In tg271Q-300 mice, injections of PRO (15 mg/kg, i.p.) eliminates startle-induced tremor episodes without producing sedation. Despite the clear connection between hyperekplexia and specific GlyR mutations, patients are commonly treated with benzodiazepines (Stewart et al., 2002; Zhou et al., 2002) that indirectly target the disease by enhancing GABA type A receptor $\left(G_{A B A} R\right)$ activity. The results presented here suggest that strategies that enhance mutant GlyR responses may provide alternative, rational treatments for hyperekplexic patients.

\section{Materials and Methods}

Materials. $\beta$-Alanine was obtained from Merck (Darmstadt, Germany); all other chemicals were obtained from Sigma (Deisenhofen, Germany). For oocyte experiments, reagent-grade PRO (Tocris/Biotrend, Cologne, Germany) was dissolved in dimethylsulfoxide to form a stock solution of $1 \mathrm{~m}$ PRO before being added to buffer. The final dimethylsulfoxide concentration was $<0.05 \%(\mathrm{v} / \mathrm{v})$, and had no significant effect on glycineinduced currents. For animal experiments, a clinical formulation of $1 \%$ $(w / v)$ PRO in soybean emulsion (Fresenius AG; Bad Homburg, Germany) was used.

Expression of mutant cDNAs in oocytes and mice. Human wt $\alpha_{1}$, $\alpha_{1}(\mathrm{R} 271 \mathrm{Q}), \alpha_{1}(\mathrm{R} 271 \mathrm{~L})$, and $\alpha_{1}(\mathrm{R} 271 \mathrm{~K})$ GlyR subunit cDNAs subcloned into the pRc/cytomegalovirus expression vector (Langosch et al., 1994) were used as templates for in vitro transcription of wt and mutant GlyR subunit cRNAs (mCAP kit; Invitrogen, Groningen, The Netherlands). Xenopus oocytes were prepared and injected with GlyR subunit cRNAs using established techniques (Langosch et al., 1994). For in vivo experi- ments, the heterozygous tg271Q-300 transgenic mouse line was bred and handled as described previously (Becker et al., 2002).

Electrophysiological recordings. Thirty-six hours after injection, two-electrode voltage clamp recordings were performed on oocytes held at a membrane potential of $-70 \mathrm{mV}$. While determining concentration-effect relationships, the oocyte was continuously perfused with either frog Ringer's solution or frog Ringer's plus PRO. Frog Ringer's solution contained the following (in mM): $115 \mathrm{NaCl}, 1 \mathrm{KCl}$, $1.8 \mathrm{CaCl}_{2}$, and 10 HEPES, pH 7.2. Agonists ( \pm PRO) were hand-applied to the oocyte chamber with a pipette. Osmotic controls with up to $500 \mathrm{~mm}$ sucrose produced no currents. Parameter values for agonist $\mathrm{EC}_{50}, n_{\mathrm{H}}$ (the Hill coefficient), and $\epsilon$ (relative efficacy: $I_{\text {max,test con }}{ }^{-}$ dition $/ I_{\text {max }, \text { Gly }}$, where the test condition is either a partial agonist or an agonist + PRO) were obtained from fitting individual experiments with the following equation: $I / I_{\max , \text { Gly }}=$ $\epsilon^{\star}\left(D^{\mathrm{nH}}\right) /\left[\left(\mathrm{EC}_{50}{ }^{\mathrm{nH}}\right)+\left(D^{\mathrm{nH}}\right)\right]$, where $D$ represents the concentration of agonist, and $I_{\max , \text { Gly }}$ corresponds to the maximal response obtained with glycine in the absence of PRO.

Behavioral analysis of transgenic mice. Drugnaive animals were injected intraperitoneally with 10 or $15 \mathrm{mg} / \mathrm{kg}$ doses of a $1 \%$ (w/v) emulsion of PRO (Fresenius AG, Bad Homburg, Germany), and righting reflexes and pain sensitivities were monitored at $0,3,8$, and $30 \mathrm{~min}$ after injection as described previously (Becker et al., 2000, 2002). At each time point, righting times were measured three times per side, and the data were pooled with the experimental results from 11 animals. Tremor was recorded as described previously (Becker et al., 2002). Pain sensitivity was determined by a hot-plate assay: animals were placed on a $52^{\circ} \mathrm{C}$ surface, and the time was measured until a paw lift or paw lick. All procedures were approved by the animal care committee and were in accordance with the German law on animal experimentation.

For all analyses, values are given as means \pm SEM. Statistical significance was determined at the $p<0.05$ and $p<0.01$ levels using Student's $t$ test.

\section{Results}

\section{PRO increases the agonist affinities of recombinant wt} $\alpha_{1}$ GlyRs

In oocytes expressing wt $\alpha_{1}$ GlyR subunits, 3-5 sec applications of $1 \mathrm{~mm}$ glycine produced maximal peak responses $\left(I_{\text {max,Gly }}\right)$ that returned to baseline during agonist washout. Oocytes were allowed to recover for at least $30 \mathrm{sec}$ between drug applications, and were periodically challenged with submaximal test concentrations of glycine to ensure that no response rundown had occurred. After establishing the $I_{\text {max,Gly }}$ for each oocyte, PRO was added to the perfusion medium, and the oocytes were preincubated in the anesthetic for $2 \mathrm{~min}$. This preincubation period with PRO alone caused no change in the holding current (e.g., no GlyR activation), but when co-applied with a submaximal concentration of glycine (i.e., $50 \mu \mathrm{M}$ ), PRO enhanced the response amplitude in a dose-dependent manner. PRO concentrations as low as $1 \mu \mathrm{M}$ already produced potentiation (Fig. $1 A$ ), with maximal potentiation being seen at $0.5 \mathrm{~mm}$ PRO (data not shown). In contrast, PRO had no effect on $\alpha_{1}$ GlyR response amplitudes at saturating concentrations of glycine (Fig. $1 B$ ). 
Table 1. Agonist response parameters obtained in two-electrode voltage-clamp experiments.

\begin{tabular}{|c|c|c|c|c|c|c|c|c|c|c|}
\hline \multirow[t]{3}{*}{ Fitted parameter } & \multicolumn{6}{|l|}{$\alpha_{1}$ GlyRs } & \multicolumn{4}{|c|}{$\alpha_{1}(\mathrm{R} 271 \mathrm{~K})$ GlyRs } \\
\hline & \multicolumn{2}{|l|}{ Glycine $(n=8)$} & \multicolumn{2}{|c|}{$\beta$-Alanine $(n=5)$} & \multicolumn{2}{|l|}{ Taurine $(n=3)$} & \multicolumn{2}{|c|}{ Glycine $(n=5)$} & \multicolumn{2}{|c|}{$\beta$-Alanine $(n=5)$} \\
\hline & $-P R O$ & +PRO & $-P R O$ & $+\mathrm{PRO}$ & $-P R O$ & $+\mathrm{PRO}$ & $-P R O$ & $+\mathrm{PRO}$ & $-P R O$ & +PRO \\
\hline $\mathrm{EC}_{50}(\mathrm{~mm})$ & $0.39 \pm 0.05$ & $0.037 \pm 0.008^{*}$ & $2.02 \pm 0.21$ & $0.09 \pm 0.02^{*}$ & $4.51 \pm 1.96$ & $0.14 \pm 0.01$ & $2.05 \pm 0.07$ & $0.18 \pm 0.05^{*}$ & $5.77 \pm 1.38$ & $0.57 \pm 0.16^{*}$ \\
\hline $\begin{array}{l}\mathrm{E}_{50} \text { ratio } \\
\epsilon\end{array}$ & $\begin{array}{l}10.5 \\
0.95 \pm 0.01\end{array}$ & $0.93 \pm 0.02$ & $\begin{array}{l}22.5 \\
0.67 \pm 0.05\end{array}$ & $0.92 \pm 0.02^{*}$ & $\begin{array}{l}32.0 \\
0.23 \pm 0.04\end{array}$ & $0.90 \pm 0.04^{*}$ & $\begin{array}{l}71.6 \\
0.96 \pm 0.01\end{array}$ & $4.1 \pm 0.46^{*}$ & $\begin{array}{l}10.2 \\
0.12 \pm 0.05\end{array}$ & $1.9 \pm 0.47^{*}$ \\
\hline$\epsilon$ ratio & 1.0 & & 1.4 & & 3.9 & & 4.3 & & 15.8 & \\
\hline
\end{tabular}

$\mathrm{EC}_{50}$ and $\epsilon$ values were averaged from curve fits of individual experiments and are expressed as means \pm SEM. *Different by $p<0.05$ from the agonist-alone condition. + PRO, Responses recorded after 2 min of preincubation with $0.5 \mathrm{~mm}$ PRO.

Figure $1 C$ shows pooled $\alpha_{1}$ GlyR concentration-effect data for multiple glycine concentrations in the presence and absence of $0.5 \mathrm{~mm}$ PRO. When normalized versus the $I_{\max , \mathrm{Gly}}$ for each oocyte, PRO potentiation appears as a parallel, leftward shift of the glycine concentration-effect curve. Parameter values from curve fits of the data (Table 1) indicate that $0.5 \mathrm{~mm}$ PRO decreased the glycine $\mathrm{EC}_{50}$ value by 10 -fold at $\alpha_{1}$ GlyRs, but had no effect on the maximal current elicited by glycine (relative efficacy $\epsilon)$ or Hill slope $\left(n_{\mathrm{H}}\right)$ value (data not shown).

PRO restores glycine relative efficacy at hyperekplexic GlyRs Previously, we have shown that glycine has increased $\mathrm{EC}_{50}$ values and smaller maximal responses at GlyRs carrying $\alpha_{1}(\mathrm{R} 271)$ substitutions (Langosch et al., 1994). In contrast with wt $\alpha_{1}$ GlyRs, high concentrations of glycine $(\geq 100 \mathrm{~mm})$ are required to achieve the $I_{\max , \text { Gly }}$ at $\alpha_{1}(\mathrm{R} 271 \mathrm{Q})$ and $\alpha_{1}(\mathrm{R} 271 \mathrm{~L})$ GlyRs (Langosch et al., 1994). Similar to wt $\alpha_{1}$ GlyRs, low concentrations of PRO $(1 \mu \mathrm{M})$ were able to potentiate glycine-induced responses at $\alpha_{1}(\mathrm{R} 271 \mathrm{Q})$ GlyRs at low receptor occupancy $\left(<\mathrm{EC}_{10}\right)$ (Fig. $\left.1 A\right)$. However, in contrast with $\alpha_{1}$ wt GlyRs, on addition of $0.5 \mathrm{~mm}$ PRO to the perfusion medium $100 \mathrm{~mm}$ glycine produced responses at $\alpha_{1}(\mathrm{R} 271 \mathrm{Q})$ and $\alpha_{1}(\mathrm{R} 271 \mathrm{~L})$ GlyRs that were 26- and 19 -fold larger than the $I_{\max , G l y}$, respectively (Fig. $1 B$ ) (data not shown). Figure $1 B$ also shows that in the absence of PRO, $100 \mathrm{~mm}$ glycine elicited only low-amplitude responses in $\alpha_{1}$ (R271Q) GlyRs, a fact that impedes precise dose-response analysis. Therefore, to analyze in more detail the effect of PRO at GlyRs carrying a mutation at position 271, we chose the isofunctional mutation $\alpha_{1}(\mathrm{R} 271 \mathrm{~K})$, which displays less-pronounced effects on agonist affinity and maximal inducible currents (Langosch et al., 1994). After the addition of $0.5 \mathrm{~mm}$ PRO to the perfusion medium, 10 mM glycine produced maximal responses at $\alpha_{1}(\mathrm{R} 271 \mathrm{~K})$ GlyRs that also were significantly larger than the $I_{\max , \text { Gly }}($ Fig. $1 B$ ), indicating that PRO has qualitatively similar effects at all $\alpha_{1}$ (R271) GlyR mutants. In different concentration-effect experiments (Fig. 1C), PRO was found to potentiate the responses of $\alpha_{1}(\mathrm{R} 271 \mathrm{~K})$ GlyRs to both submaximal and maximal concentrations of glycine. Fitted parameter values (Table 1) indicate that PRO decreased the glycine $\mathrm{EC}_{50}$ value by 12 -fold, increased glycine $\epsilon$ values by 4 -fold, and had no significant effect on glycine $n_{\mathrm{H}}$ values at $\alpha_{1}(\mathrm{R} 271 \mathrm{~K})$ GlyRs. Together, these results indicate that hyperekplexic mutant GlyRs have small maximal responses, but are potently potentiated by $\mathrm{PRO}$ at saturating concentrations of glycine.

PRO improves partial agonist relative efficacies at $w t$ and hyperekplexic GlyRs

At GABA ${ }_{A}$ Rs, PRO has been demonstrated to increase the $\epsilon$ value of a partial agonist (O'Shea et al., 2000). We therefore tested whether PRO might also improve the $\epsilon$ values of partial agonists at GlyRs. Neither $\beta$-alanine nor taurine produces detectable cur-

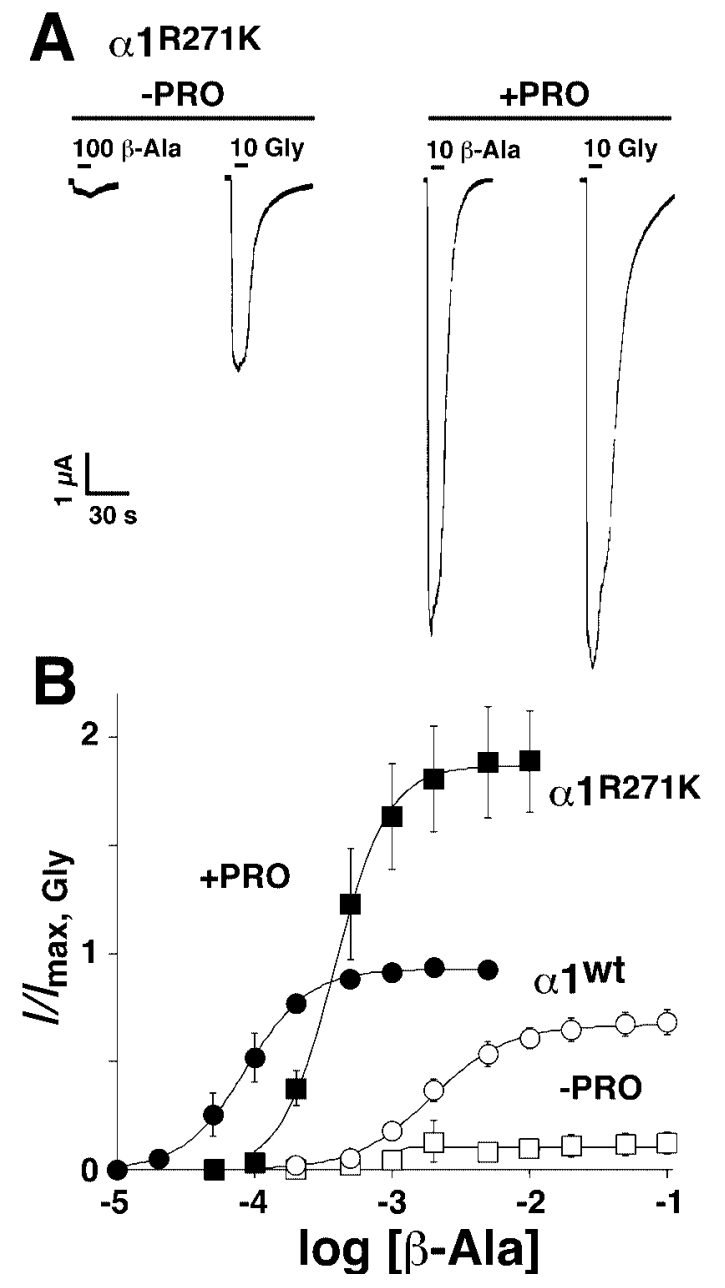

Figure 2. PRO enhances the maximal responses of wt and hyperekplexic $\alpha_{1}$ GlyRs to partial agonists. $A, \beta$-Alanine and glycine-induced responses recorded from oocytes expressing $\alpha_{1}(\mathrm{R} 271 \mathrm{~K})$ GlyRs in the absence (-PR0) or presence (+PRO) of bath-applied PRO (0.5 mm). Bars indicate the duration of agonist application; agonist concentrations are given in mм. $B$, Pooled dose-response curves for $\beta$-alanine for $\alpha_{1}$ wt (circles) and $\alpha_{1}$ (R271K) (squares) GlyRs in the absence (open symbols) and presence (filled symbols) of $0.5 \mathrm{~mm}$ PRO, respectively. Data are normalized to $I_{\text {max,Gly }}$ obtained with saturating glycine concentrations $(n=5)$.

rents at $\alpha_{1}(\mathrm{R} 271 \mathrm{~L})$ or $\alpha_{1}(\mathrm{R} 271 \mathrm{Q})$ GlyRs (Laube et al., 1995). At $\alpha_{1}(\mathrm{R} 271 \mathrm{~K})$ GlyRs, saturating concentrations of $\beta$-alanine generated $12 \pm 5 \%$ of the $I_{\max , \text { Gly }}$ (Table 1 ). At these receptors, the addition of $0.5 \mathrm{~mm}$ PRO resulted in responses to $10 \mathrm{~mm} \beta$-alanine that were larger than the $I_{\max , G l y}$ without PRO and comparable with the maximal inducible glycine currents in the presence of PRO (Fig. 2A). On average, $\beta$-alanine plus $0.5 \mathrm{~mm}$ PRO elicited responses that were roughly twofold larger than the $I_{\max , \text { Gly }}$ (Fig. $2 B$ ). This marked enhancement was reflected by a 16 -fold in- 
crease in the $\epsilon$ value for $\beta$-alanine and a 10 -fold decrease in the $\mathrm{EC}_{50}$ (Table 1). At wt GlyRs, saturating concentrations of $\beta$-alanine and taurine produced responses that typically reached $67 \pm 5$ and $23 \pm 4 \%$, respectively, of the $I_{\max , \text { Gly }}$ (Table 1 ) (Laube et al., 1995). PRO (0.5 mM) potentiated the maximal responses of both partial agonists to nearly the $I_{\max , \text { Gly }}$. When comparing the effects of PRO on a range of $\beta$-alanine and taurine concentrations, PRO produced nonparallel, leftward shifts of both concentration effect curves (Fig. 2 B). Table 1 summarizes these results: PRO decreased the $\mathrm{EC}_{50}$ values of $\beta$-alanine (23-fold) and taurine (32-fold), increased the $\epsilon$ values (1.4- and 4-fold, respectively), and significantly increased the $n_{\mathrm{H}}$ values of both agonists at $\alpha_{1}$ GlyRs (data not shown). Therefore, assuming that the small responses seen with glycine, $\beta$-alanine, and taurine at R271substituted GlyRs (Table 1) contribute to the small glycinergic IPSC amplitudes observed in spinal cord slices from tg271Q-300 mice (Becker et al., 2002), we wondered whether PRO could be used to improve the neuromotor deficits of this animal hyperekplexia model.

\section{PRO reduces the startle responses and tremor episodes of $\operatorname{tg} 271$ Q-300 mice}

Compared with wt mice, transgenic hyperekplexic mice expressing human $\alpha_{1}(\mathrm{R} 271 \mathrm{Q})$ GlyRs show exaggerated jerks and jumps after a sudden noise or touch (Becker et al., 2002). Although a high inter-animal variability of these startle responses makes the behavior difficult to standardize and quantify, the differences in neuromotor performance between the two mouse lines are obvious to a trained observer. Notably, several minutes after an injection of $1 \%$ PRO (15 mg/kg, i.p.), the abnormal startle reflex of R271Q mice disappeared, and responses became indistinguishable from those of wt mice. This effect was transient, and the exaggerated startle response returned within $30 \mathrm{~min}$ after injection.

To further quantify the "anti-hyperekplexic" properties of $\mathrm{PRO}$, we also measured its effect on handling-induced convulsive episodes. Tg271Q-300 animals show both spontaneous and handling-induced muscle tremors that occur with the same periodic frequency $(\sim 25-30 \mathrm{~Hz})$ as in startled hyperekplexic patients (Stayer and Meinck, 1998; Becker et al., 2002). Here, we conducted similar studies on tg271Q-300 mice both before and after an injection of PRO (15 mg/kg, i.p.). Figure $3 A$ shows segments from a typical experiment, obtained by suspending a mouse from an electromechanical transducer by the tail. After an unexpected stimulus or handling, untreated tg271Q-300 mice often experienced prolonged tremor episodes (Fig. $3 A$ ). In mutants treated with PRO, the same stimulus produced no such tremors (Fig. $3 A$ ). Between tremor episodes, both untreated and PRO-treated transgenic mice resembled wt mice whose muscle tone was not changed on PRO injection (Fig. 3A). Like relief from the exaggerated startle response, the effect of PRO on tg271Q-300 mice was temporary, and the hyperekplexic phenotype returned within 30 min after injection.

To exclude the possibility that the beneficial effects of PRO were attributable to oversedation, we tested tg271Q-300 mice using two classic behavioral assays for anesthesia. We checked for differences in pain sensitivity by placing the animal's foot on a hot plate and measuring the time required for a paw lift or paw lick. Before injection, mice showed $20 \mathrm{sec}$ paw-lift latencies (19.6 \pm $5.2 \mathrm{sec})$ that remained unchanged $3 \mathrm{~min}(16.3 \pm 4.9 \mathrm{sec})$ and 8 min $(19.5 \pm 9.4 \mathrm{sec}$ ) after a $15 \mathrm{mg} / \mathrm{kg}$ dose of PRO (Fig. $3 B$ ). Second, the time required for the mice to right themselves after being rolled onto their backs was measured before and after the
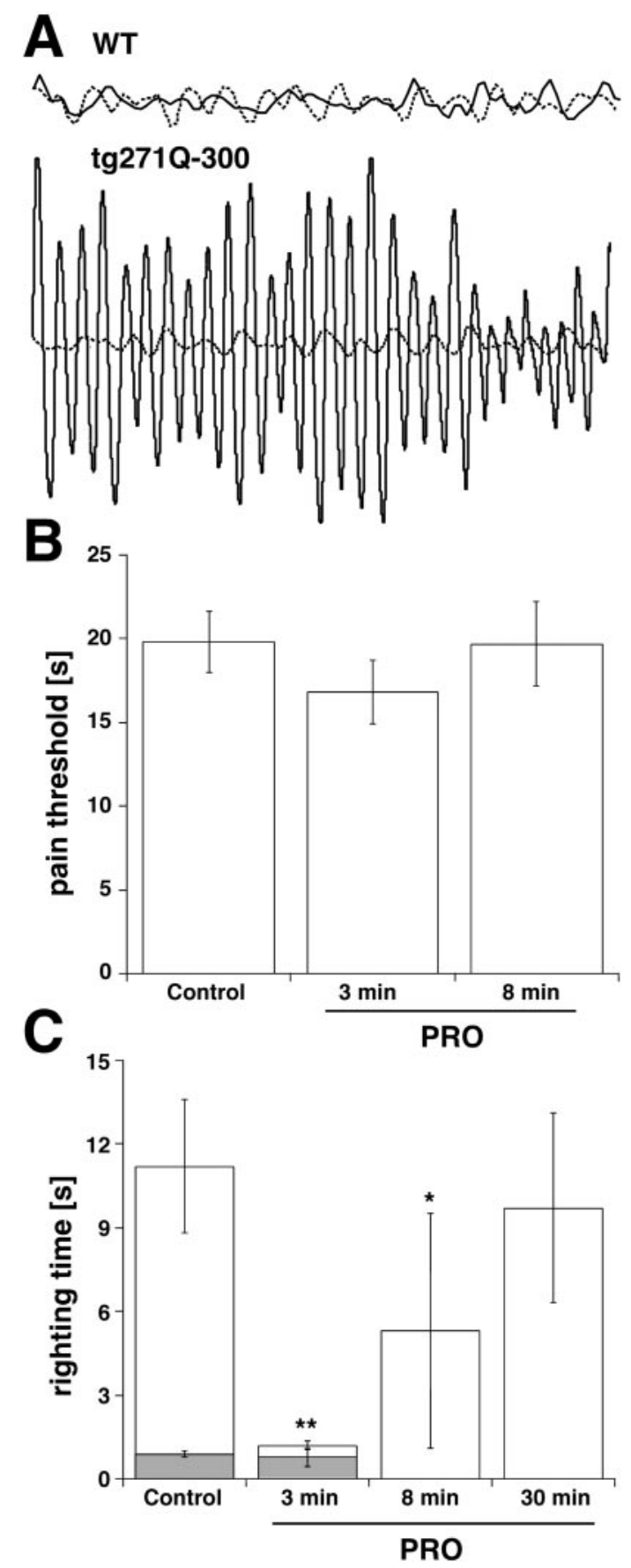

Figure 3. Behavioral effects of PRO in tg2710-300 mice. A, PRO quiets tremor in hyperekplexic mice. Shown are typical 1 sec recordings obtained from wt and tg2710-300 mice suspended by the tail from a mechanical transducer. In the upper traces, wt mice show no tremor activity before (solid line) and $3 \mathrm{~min}$ after (dotted line) a $15 \mathrm{mg} / \mathrm{kg}$, i.p., injection of PRO. In the lower traces, untreated tg2710-300 mice show high-frequency (25-30 Hz) tremors (solid line) that disappear 3 min after treatment with PRO. B, Acute pain responses to thermal stimuli in the hot-plate test. No significant differences in the latencies of withdrawal from thermal stimuli were observed between PRO-treated and control mice 3 and $8 \mathrm{~min}$, after the intraperitoneal injection of $15 \mathrm{mg} / \mathrm{kg}$ PRO when using the hot-plate test $(n=11)$. C, PRO shortens the righting times of tg271-300 mice (white columns) without affecting those of wt mice (gray columns). Righting time latencies represent the time required for achieving a standing position either before (Control), or 3, 8, or $30 \mathrm{~min}$ after $15 \mathrm{mg} / \mathrm{kg}$, i.p., injections of PRO. ${ }^{*} p<0.05$ versus controls; ${ }^{* *} p<0.01$ versus controls $(n=11)$. 
injection of PRO. As outlined below (Fig. 3C), instead of producing an increase in righting time associated with sedation, PRO significantly decreased the righting time of tg271Q-300 mice. In summary, the doses of PRO used here to treat hyperekplexia were too low to induce anesthesia, and produced no observable sedation in tg271Q-300 mice.

\section{PRO restores normal locomotor coordination to $\operatorname{tg} 271 \mathrm{Q}-300$ mice}

In addition to the exaggerated startle responses and muscle rigidity that are diagnostic features of hyperekplexia in humans, tg271Q-300 mice display two motor defects that distinguish them from wt mice: poorly coordinated movements and significantly slower righting times. The first is easily monitored as an uncoordinated walking behavior, and a clasping of the hindfeet when mutant animals are picked up by their tails (Becker et al., 2002). Surprisingly, tail-suspended mutants treated with PRO balanced their position by spreading out the legs and showed wt-like walking behavior (data not shown). Furthermore, compared with wt mice that normally achieve a standing position within less than a second after being rolled onto their backs, untreated tg271Q-300 mice showed mean righting times of $\sim 10 \mathrm{sec}$ (Fig. 3C). Notably, $3 \mathrm{~min}$ after a $15 \mathrm{mg} / \mathrm{kg}$ injection of PRO, mutant mice showed a mean righting time of $\approx 1 \mathrm{sec}$, similar to those found for wt mice (Fig. 3C). After reaching an upright position, PRO-treated mice walked and moved normally. These improvements were temporary: $8 \mathrm{~min}$ after injection, the righting times had increased to 5 $\mathrm{sec}$, and by $30 \mathrm{~min}$ the slow righting behavior had returned. To summarize, hyperekplexic mice treated acutely with PRO showed locomotor abilities that closely resembled those of untreated wt animals.

\section{Discussion}

Previous studies of GlyR allosteric modulation typically have focused on the enhancement of responses to submaximal concentrations of glycine (for review, see Laube et al., 2002). However, different reports suggest that saturating concentrations of agonist may produce better approximations of electrically evoked IPSCs in spinal cord and brainstem (Clements, 1996; Laube, 2002). Therefore, the ability of PRO to enhance the maximal responses of GlyRs carrying $\alpha_{1}(\mathrm{R} 271)$ hyperekplexia mutations has relevance for glycinergic inhibition in hyperekplexic animals and patients.

As summarized in Table 1, PRO produced a fourfold increase in the $\epsilon$ value of glycine at $\alpha_{1}(\mathrm{R} 271 \mathrm{~K})$ GlyRs, an improvement that should fully restore the threefold reductions observed in the glycinergic IPSC amplitudes of tg271Q-300 mice (Becker et al., 2002). Of the agonist-receptor combinations tested, PRO produced the largest $\epsilon$ ratio (16-fold) for $\beta$-alanine at $\alpha_{1}(\mathrm{R} 271 \mathrm{~K})$ GlyRs, but PRO may produce even larger enhancements of glycine responses at $\alpha_{1}(\mathrm{R} 271 \mathrm{~L})$ and $\alpha_{1}(\mathrm{R} 271 \mathrm{Q})$ GlyRs that could not be analyzed precisely because of the rather small responses seen in the absence of PRO. The ability of PRO to increase agonist $\epsilon$ values was not limited to mutants, because PRO converted $\beta$-alanine and taurine into full agonists at wt $\alpha_{1}$ GlyRs. The published concentrations of PRO producing GlyR potentiation show some variability, but modulating effects have consistently been seen at $\geq 1 \mu \mathrm{M}$ (Belelli et al., 1999; Hadipour-Jahromy and Daniels, 2003).

PRO also increases the apparent agonist affinities of all GlyRs tested. For example, PRO decreased the $\mathrm{EC}_{50}$ value of glycine at $\alpha_{1}(\mathrm{R} 271 \mathrm{~K})$ GlyRs by 12 -fold, producing mutant GlyRs with $\mathrm{EC}_{50}$ values lower than of unmodulated wt $\alpha_{1}$ GlyRs (Table 1). PRO had similar effects on $\beta$-alanine elicited currents, and increased its $\mathrm{EC}_{50}$ value by 10 -fold. Given that PRO had the largest effects on the $\epsilon$ value of $\beta$-alanine at $\alpha_{1}(\mathrm{R} 271 \mathrm{~K})$ GlyRs, we expected to see a larger increase in the $\mathrm{EC}_{50}$ of $\beta$-alanine. In fact, there appeared to be a loose correlation between the $\mathrm{EC}_{50}$ values and $\epsilon$ ratios for most agonist-receptor combinations in the presence of PRO (Table 1). Our relatively slow agonist application speed and the decision to normalize all responses to $I_{\max , \text { Gly }}$ clearly influenced this relationship, but it is tempting to propose a mechanistic link between these two parameters (Colquhoun, 1998). We are currently investigating the kinetic properties of $\alpha_{1}(\mathrm{R} 271 \mathrm{~K})$ GlyRs to determine if PRO influences agonist binding and/or channel gating.

In agreement with previous reports on wt GlyRs (Belelli et al., 1999; Hadipour-Jahromy and Daniels, 2003), low micromolar concentrations of PRO were effective in increasing the maximal response of the $\alpha_{1}(\mathrm{R} 271 \mathrm{Q})$ GlyR to glycine in oocytes (Fig. 1A). We therefore reasoned that PRO might also increase the amplitudes of IPSCs in mice expressing a human $\alpha_{1}(\mathrm{R} 271 \mathrm{Q})$ GlyR transgene. Tg271Q-300 mice show a number of behavioral phenotypes that make them an excellent model for hereditary hyperekplexia in humans (Becker et al., 2002). In response to sudden stimuli, mutant mice show exaggerated startle responses, muscle rigidity, and tremor episodes that mimic the behavior seen with affected patients (Stayer and Meinck, 1998). In addition to these reflexive deficits, mutant mice also show a reduction in muscle coordination associated with human hyperekplexia.

Shortly after an injection of PRO (15 mg/kg, i.p.), tg271Q-300 mice showed temporary abatements in tremor episodes and significantly improved righting responses that were consistent with a reduction in the hyperekplexia phenotype. More subjectively, the startle responses and locomotor coordination of PRO-treated mutant mice were indistinguishable from the behavior of $\mathrm{wt}$ mice. Presumably because of the improvement in muscle coordination, PRO-treated mice also showed normalization of righting times. Despite the well-known ability of PRO to enhance $\mathrm{GABA}_{\mathrm{A}} \mathrm{R}$ activity (Belelli et al., 1999; Jurd et al., 2003), causing anesthesia on higher-dose intraperitoneal injection (e.g., $140 \mathrm{mg} /$ $\mathrm{kg}$; Irifune et al., 2003), the low doses of PRO used in this study did not appear to have anesthetic or sedative effects. Because a large reduction for $\mathrm{GABA}_{\mathrm{A}} \mathrm{R}$-mediated inhibitory transmission was also found in the tg271Q-300 mice (Becker et al., 2002) and mice carrying a point mutation in the $\beta_{3}$ subunit of the $\mathrm{GABA}_{\mathrm{A}} \mathrm{R}$ display a reduction in the loss of the righting reflex after PRO treatment (Jurd et al., 2003), a pleiotropic effect of subanesthetic $\mathrm{PRO}$ concentrations at both $\mathrm{GABA}_{\mathrm{A}}$ Rs and GlyRs cannot be excluded. However, because PRO concentrations as low as $1 \mu \mathrm{M}$ potentiated GlyR responses in oocytes, PRO clearly can act as an in vivo potentiator of mutant GlyRs. Future behavioral tests will be required to determine the effects of $\mathrm{PRO}$ on higher cognitive functions (e.g., learning and memory, attention) and brain metabolism in mice, but PRO appears to be a promising lead compound for the rational design of drugs that target human hyperekplexia.

\section{References}

Andermann F, Keene DL, Andermann E, Quesney LF (1980) Startle disease or hyperekplexia: future delineation of the syndrome. Brain 103:985-997.

Becker L, Hartenstein B, Schenkel J, Kuhse J, Betz H, Weiher H (2000) Transient neuromotor phenotype in transgenic spastic mice expressing low levels of glycine receptor beta-subunit: an animal model of startle disease. Eur J Neurosci 12:27-32.

Becker L, von Wegerer J, Schenkel J, Zeilhofer HU, Swandulla D, Weiher H (2002) Disease-specific human glycine receptor $\alpha 1$ subunit causes hy- 
perekplexia phenotype and impaired glycine- and $\mathrm{GABA}_{\mathrm{A}}$-receptor transmission in transgenic mice. J Neurosci 22:2505-2512.

Belelli D, Pistis M, Peters JA, Lambert JJ (1999) The interaction of general anaesthetics and neurosteroids with $\mathrm{GABA}_{\mathrm{A}}$ and glycine receptors. Neurochem Int 34:447-452.

Clements JD (1996) Transmitter timecourse in the synaptic cleft: its role in central synaptic function. Trends Neurosci 19:163-171.

Colquhoun D (1998) Binding, gating, affinity and efficacy: the interpretation of structure-activity relationships for agonists and of the effects of mutating receptors. Br J Pharmacol 125:924-947.

Floeter MK, Hallet M (1993) Glycine receptors: a startling connection. Nat Genet 5:319-320.

Hadipour-Jahromy M, Daniels S (2003) Binary combinations of propofol and barbiturates on human $\alpha 1$ glycine receptors expressed in Xenopus oocytes. Eur J Pharmacol 477:81-86.

Irifune M, Takarada T, Shimizu Y, Endo C, Katayama S, Dohi T, Kawahara M (2003) Propofol-induced anaesthesia in mice is mediated by gammaaminobutyric acid-A and excitatory amino acid receptors. Anaesth Analg 97:424-429.

Jurd R, Arras M, Lambert S, Drexler B, Siegwart R, Crestani F, Zaugg M, Vogt KE, Ledermann B, Antkowiak B, Rudolph U (2003) General anesthetic actions in vivo strongly attenuated by a point mutation in the GABA(A) receptor beta3 subunit. FASEB J 17:250-252.

Langosch D, Laube B, Rundstrom N, Schmieden V, Bormann J, Betz H (1994) Decreased agonist affinity and chloride conductance of mutant glycine receptors associated with human hereditary hyperekplexia. EMBO J 13:4223-4228.

Laube B (2002) Potentiation of inhibitory glycinergic neurotransmission by $\mathrm{Zn}^{2+}$ : a synergistic interplay between presynaptic $\mathrm{P} 2 \mathrm{X}_{2}$ and postsynaptic glycine receptors. Eur J Neurosci 16:1025-1036.

Laube B, Langosch D, Betz H, Schmieden V (1995) Hyperekplexia mutations of the glycine receptor unmask the inhibitory subsite for betaamino-acids. NeuroReport 6:897-900.

Laube B, Maksay G, Schemm R, Betz H (2002) Modulation of glycine receptor function: a novel approach for therapeutic intervention at inhibitory synapses? Trends Pharmacol Sci 23:519-527.

O'Shea SM, Wong LC, Harrison NL (2000) Propofol increases agonist efficacy at the GABA(A) receptor. Brain Res 852:344-348.

Schofield PR (2002) The role of glycine and glycine receptors in myoclonus and startle syndromes. Adv Neurol 89:263-274.

Shiang R, Ryan SG, Zhu YZ, Hahn AF, O'Connell P, Wasmuth JJ (1993) Mutations in the alphal subunit of the inhibitory glycine receptor cause the dominant neurologic disorder, hyperekplexia. Nat Genet 5:351-358.

Stayer C, Meinck HM (1998) Stiff-man syndrome: an overview. Neurologia 13:83-88.

Stewart WA, Wood EP, Gordon KE, Camfield PR (2002) Successful treatment of severe infantile hyperekplexia with low-dose clobazam. J Child Neurol 17:154-156.

Zhou L, Chillag KL, Nigro MA (2002) Hyperekplexia: a treatable neurogenetic disease. Brain Dev 24:669-674. 